\title{
La desconfianza en los sistemas de impartición de justicia mexicana, prospección y evaluación desde la perspectiva del Conflict Analysis Tipology (CAT)
}

\author{
The distrust in systems of impartition of mexican justice, \\ prospection and evaluation through the perspective of the Conflict \\ Analysis Tipology (CAT)
}

Recibido: 20-04-2021 | Aceptado: 08-07-2021

Jazmín Flores-Montes*

*https://orcid.org/0000-0001-8197-6782

México

\section{Resumen}

Los niveles de percepción de confianza en México son preocupantes, esto implica que se desconozcan los beneficios que posee como valor intangible para que sea vista como un factor imprescindible propio del ser humano y este pueda ser capaz de resolver sus propios conflictos; los que surgen de su relación con otros sujetos en el entorno social; y aquellos que versan ante las instituciones del Estado encargadas de impartir justicia tradicional y alterna como la mediación.

El presente artículo explica la aplicación del instrumento cualitativo Conflict Analysis Tipology (CAT) de Josep Redorta, en la investigación denominada "Los elementos generadores de Confianza como Valor intangible en la mediación" que permite la identificación del tipo de conflicto que puede suscitarse, a través de la tipicación de patrones dominantes, los cuales sustentan y otorgan rigor científico a la problemática de estudio planteada, además, de que nos permitirá centrar la intervención adecuada para la transformación y solución del conflicto detectado.

Palabras clave: Confianza, Valor Intangible, Conflict Analysis Tipology (Cat), Mediación, Conflicto.

\section{Abstract}

The levels of perception of trust in Mexico are worrying, this implies that the benefits it possesses as

\section{Cómo citar}

Flores-Montes, J. La desconfianza en los sistemas de impartición de justicia mexicana, prospección y evaluación desde la perspectiva del Conflict Analysis Tipology (CAT). MSC Métodos De Solución De Conflictos. Recuperado a partir de https://revistamsc.uanl.mx/index.php/m/article/ view/6 
an intangible value are unknown so that it is seen as an essential factor of the human being and this may be able to resolve its own conflicts; those that arise from their relationship with other subjects in the social environment; and those that deal with the State institutions in charge of imparting traditional and alternative justice such as mediation.

This article explains the application of the qualitative instrument Conflict Analysis Typology (CAT) by Josep Redorta, in the research called "The elements that generate Trust as an intangible value in mediation" that allows the identification of the type of conflict that may arise, through of the typification of dominant patterns, which sustain and grant scientific rigor to the problem of study raised, in addition, that it will allow us to focus the appropriate intervention for the transformation and solution of the detected conflict.

Key words: Confidence, Intangible Value, Conflict Analysis Tipology (Cat), Mediation, Conflict.

\section{INTRODUCCIÓN}

Las instituciones del sistema judicial mexicano cuya función primordial consiste en proteger el Derecho humano de acceso a la justicia a través de la impartición de justicia mediante sus diversas instancias propias o delegadas hacia la coadyubación del mismo propósito, de tal manera que en la actualidad se han visto desfavorecidas con índices desfavorables y alarmantes, cifras negras, que indican que algo no está funcionando correctamente o que no está llevándose a cabo de forma oportuna lo que impiden su institucionalización.

En ese orden de ideas se pretende mostrar al lector la identificación del conflicto a través del CAT, así como el proceso de identificación de la tipología del conflicto planteado, lo que permitirá poder gestionarlo y transformarlo con las herramientas que aporta Josep Redorta, así como con el postulado de estrategia de intervención que ofrece la confianza como valor intangible.

\section{JUSTIFICACIÓN Y PLANTEAMIENTO DEL PROBLEMA PARA LA APLICACIÓN DEL CONFLICT ANALYSIS TIPOLOGY (CAT)}

Antes de comenzar con el análisis del instrumento Conflict Analysis Tipology (CAT), el cual permitirá identificar los patrones dominantes en nuestro conflicto, es de suma importancia puntualizar en primera instancia el identificar el conflicto y contextualizar la problemática de estudio para facilitar su comprensión, y en segunda, justificar el porqué de su existencia, para finalizar delineando su alcance.

Según el Latinobárometro 1995-2015 Opinión Pública Latinoamericana (2015, pág. 4) los niveles de confianza en América Latina son preocupantes, pues declaran que existe una pseudocrisis de confianza, ya que, en comparación con países de otras regiones del mundo, estos tienen índices más altos de confianza interpersonal y hacia las instituciones del Estado. 
Lo anterior es visto en el contexto latinoamericano, en el ámbito de México como nación, la reseña (Informe País sobre la calidad de la ciudadanía en México, 2015, pág. 2015) declara que en torno a la confianza que la ciudadanía le tiene al Poder Judicial de la Federación (autoridad encargada en México de la impartición de justicia) corresponde al $28 \%$ de los mexicanos encuestados en 2015, por lo que el resto manifiesta que no confía en las autoridades encargadas de la administración de justicia en el país, un $53.7 \%$ de percepción negativa durante el 2016, y en el 2017 obtuvo el $55.1 \%$ de no confianza hacia las autoridades del Poder Judicial. ¿Son correctos los dos últimos porcentajes y es correcto la palabra negativa y confianza?

Igualmente, en la (Encuesta Nacional sobre Cultura Política y Prácticas Ciudadanas, 2012) expresa que el $72 \%$ de los encuestados consideraba que no se podía confiar en otras personas, conjuntamente (Moreno, 2005) expresa que ese porcentaje representa un incremento frente al 10\% que respondió al año siguiente sobre esa misma cuestión en (Instituto Nacional Electoral [INE], 2015).

A partir de lo manifestado supralíneas, podemos afirmar que los niveles de percepción de confianza en las relaciones interpersonales e institucionales son preocupantes, pues no se tiene información oportuna que acredite lo contrario, implicando que esta percepción de desconocimiento de los beneficios que posee la confianza sea un factor imprescindible para que el ser humano sea capaz de resolver: sus propios conflictos; las disputas que surgen de su relación con otros sujetos en el entorno social; y aquellos que versan ante las instituciones del Estado encargadas de impartir justicia tradicional y alternativa.

Esta desconfianza complica y lacera a las instituciones de impartición de justicia, la que se ve reflejada en tres aspectos:

En primer lugar, esta gradual desconfianza, provoca que la ciudadanía, los sujetos morales y privados no asistan ante los Tribunales que imparten la Justicia Tradicional a ejercer su derecho humano de acceso a la justicia.

El segundo aspecto es de consecuencia. El hecho de que las personas, los sujetos morales y privados no confíen en las instituciones tradicionales de impartición de justicia, excluye de facto a la justicia alterna de los Métodos Alternativos de Solución de Conflictos (MASC) como una vía sencilla y rápida para la resolución de conflictos, ya que la vía de acceso o la forma de llegar a ellos sigue siendo a través de la justicia tradicional.

El último aspecto y más importante refiere entonces que, la desconfianza en las instituciones encargadas de la impartición de justicia tradicional, de la justicia alterna que ofrece la mediación pública y privada como los MASC, se siga incrementando. Esta crisis de no confianza, la cual impacta directamente en el bienestar subjetivo del ciudadano que vive en México y en la generación de sociedades pacíficas que fomenten la paz. 


\section{CONFLICT ANALYSIS TIPOLOGY (CAT) DE JOSEP REDORTA}

Ahora bien, para comprender adecuadamente cuando se enuncien las palabras patrón de conflicto dentro del CAT en líneas posteriores, se comparte la concepción que aporta (Redorta) sobre ello. En ese sentido, el autor explica que el patrón de conflicto se refiere a las conductas sociales consuetudinarias que pueden ser diferenciadas, donde existe unidad contenido de cada una y se presenta en pugnas entre personas de cualquier entorno social, pudiendo ser dual su aparición en dos o más conductas (2004, pág. 10)

Esta evolución sobre los patrones de conflicto tuvo auge en el año de 1995, ya que en ese tiempo ya existía un modelo de 5 patrones de conflicto, los cuales podían verse identificados particularmente cada uno dependiendo de las características, representando que por medio de la percepción (Redorta) pudo analizar a cada uno, además les otorgó la suficiente información para poder intervenir oportunamente en ellos. Es decir, comenzando por describir lo que ya sabemos sobre el conflicto, pero haciendo la interrogante (Redorta, 2004, págs. 9-10) que a su parecer eran irresolubles los siguientes aspectos:

1. El reconocimiento del patrón que puede llegar a seguir un conflicto, explicándolo en el sentido de su discriminación en conflictos concretos y

2. El grado de pertenencia del conflicto de cada patrón, particularmente si se mezclaba con otro.
Al mismo tiempo muestra un sinfín de definiciones de lo que es conflicto, además expone tales ideas desde la perspectiva de autores de diferentes ciencias, puntualizando aquellas palabras que son elementos que dan cabida a tales concepciones, siendo estas las más importantes a mi consideración: actividad incompatible, diferencia de intereses, disputa, dilema, contradicción, interacción hostil, intereses opuestos. Destacando el estudio que realizó Eduardo Adame en los años 1993-1996, al encontrar 79 definiciones distintas de conflicto interpersonal y ofreciendo una nueva definición: "Un proceso cognitivo-emocional en el que dos sujetos perciben fines discordantes dentro de su vínculo de interdependencia y la pretensión de solucionar sus discrepancias de poder" (Redorta, 2004, pág. 15). Por lo que el término conflicto que describe el autor es se obtiene analizando, dependiendo de la ciencia que se invoque, tendiendo su concepción ad hoc al contexto que se utilice, permitiendo ser multidisciplinar, y no sólo se encuadre en la psicología social, como primeramente se pretendía dirigir, suponiendo una autolimitación de esta, pero a la vez muy amplia para ser investigado transversalmente.

\subsection{Conflict Analysis Tipology (CAT) Apli- cado al Conflicto de la Desconfianza en los Sistemas de Impartición de Justicia Mexicana}

Contextualizado el conflicto, así como descrita la problemática planteada supralíneas, se procede a analizar el conflicto para establecer la presencia de los patrones dominantes del mismo. 
Para ello, acudimos al instrumento denominado: Conflict Analysis Tipology (CAT) elaborado por Josep Redorta, que parte de una composición de 16 patrones de conflicto que configuran las relaciones interpersonales (Redorta 2004, pág. 135), estos a su vez están subdivididos en cuatro categorías de necesidades del ser humano en la vida: 1 . Ser, es decir, ser consistente en formar nuestra propia personalidad como entes individuales y como parte de una sociedad con información, valores, identidad y autoestima; 2. Seguir, este se desprende del anterior, manifestando que debemos continuar siendo como somos en nuestro propio ser y en el entorno social como expectativas, de inadaptación, atributivo y de inhibición; 3 . Conseguir, se obtiene algo a través de la conexión al orbe, ya que nuestros anhelos nos acercan a lograr eso que queremos, se incluyen aquí los patrones de poder, legitimación, normativo e incompatibilidad personal persistente; y por último 4. Mantener, refiriéndose a que una vez que alcanzamos nuestros propósitos en la vida, debemos luchar por mantenerlos, como los patrones de conflicto de recursos escasos, de intereses, estructural y de inequidad (Redorta, 2016) y (Redorta, 2014, págs. 132-133).

También se utilizó una escala Likert instrumento que mide el grado de apreciación que va de uno a cinco, en donde uno es consenso nulo y cinco muy alto.

Algunos patrones se interrelacionan por la propia dinámica de transformación de un conflicto, haciendo que un patrón pueda convertirse en otro, y resultando que cualquier tipo de conflicto pueda encua- drarse en este modelo. Tratándose de un instrumento cualitativo que puede apreciar el nivel de pertenencia a cada patrón, derivándose que el prototipo le permita hacer esa diferencia (Redorta, 2014, pág. 6 y 9).

Estos 16 patrones se incluyen dentro de la morfología del conflicto, donde se integra lo que podrían llegar a ser los elementos condicionantes de la evolución de conflictos o factores que derivan ese conflicto de forma básica. El perfil de conflicto permite considerar aquellos elementos que siendo externos al patrón o patrones que sigue un conflicto concreto, son muy relevantes para considerar el nivel del conflicto y el conjunto de elementos clave sobre los que existe un consenso bastante amplio en la ciencia y en la actualidad (Redorta, 2014, pág. 11). Esto permite tomar decisiones para intervenir en la gestión adecuada del conflicto.

Los dos modelos de conflicto existentes en la ciencia se basan, el primero de ellos, en los que provienen de la investigación (descriptivos), y los segundos se fundamentan en la práctica derivada de la observación o de la consuetudinariedad de los que lo viven (normativos) (Redorta, 2014, pág. 3).

De tal forma que el CAT accede a que, mediante la afirmación de una oración se pueda evidenciar el alcance de un tipo de conflicto, con la finalidad de saber cuáles estrategias son adecuadas para abordarlo, cómo debemos aplicarlas para transformarlo y cómo cooperar en su la gestión, transformación y solución. 
Para la ejecución de este instrumento cualitativo, se aplicaron 30 instrumentos a ciudadanos que reconocen el conflicto planteado y a expertos en la materia, con las habilidades científicas, y prácticas de identificación de éste. A partir de la concentración de la información arrojada de dicho instrumento, se detectaron los siguientes patrones dominantes: información, valores, expectativas, normativo y estructural.

Es importante mencionar que, del total de los 16 patrones existentes, se graficaron sólo los patrones dominantes que obtuvieron la máxima puntuación, categorizando el grado de apreciación como muy alto con la puntación máxima, logrando determinar cuáles fueron los patrones dominantes del conflicto de nuestra investigación, así como aquellos otros patrones que dentro de la escala Likert (Creswell \& Plano Clark, 2007) también obtuvieron un puntaje superior al grado de apreciación medio y alto pero menor al tope indicado, como lo son los patrones de legitimación y valores.
Ahora con dicha información obtenida, se presenta la definición operativa de conflicto; el conflicto básico del cual emana la morfología del conflicto de cada patrón dominante; se desarrollarán los procesos subyacentes o puntos críticos de exploración dentro de la problemática ostentada que nos servirá para aclarar o confirmar la existencia de un patrón determinado en grado apreciable, para finalizar estableciendo las diversas estrategias que propone (Redorta, pp. 117, 120 $124,156-159)$ en su modelo para transformar los conflictos en nuestra investigación:

\section{Patrón dominante de Valores}

Este modelo relativo a la morfología de conflicto es sobre valores, en ese sentido Josep (Redorta) poner año de la cita revela que el conflicto base de este patrón dominante es de descreencia/creencia, es decir, el autor lo define en que disputamos porque mis valores o creencias fundamentales están en juego.

\section{Gráfica 1. Análisis de patrones dominantes del conflicto de la desconfianza en los sistemas de impartición de justicia mexicana.}

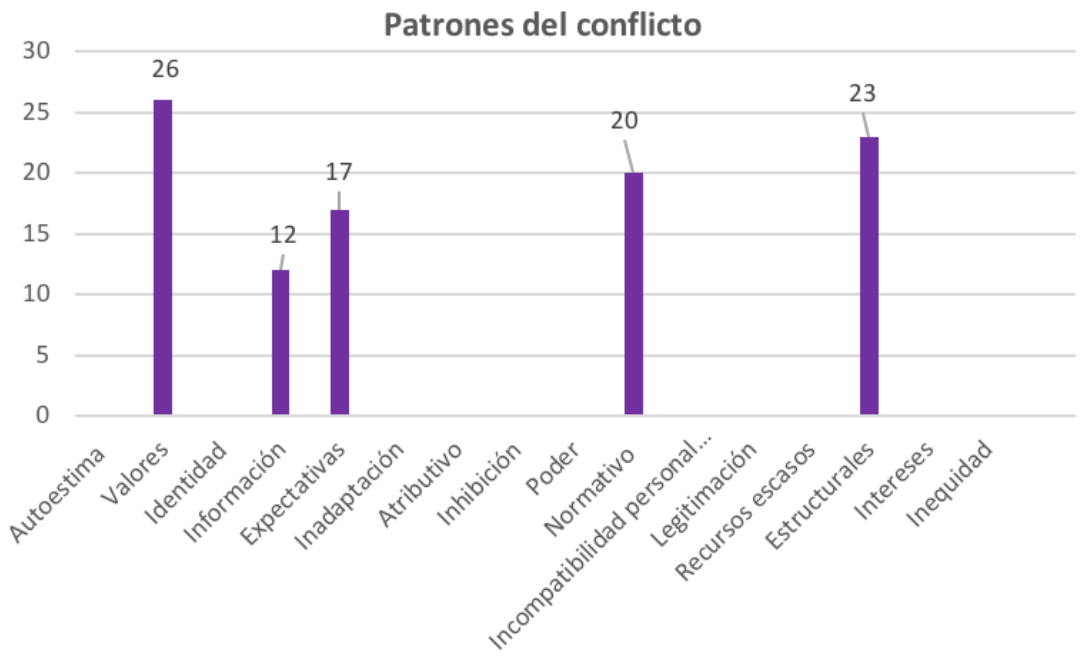

Fuente: Elaboración propia. 
De tal forma como lo manifestaron la mayor parte de los participantes del CAT en relación con los valores, se interpreta a la confianza como intangible de la mediación, de los MASC y de la justicia tradicional como el valor que está en juego y el contravalor de ésta sería la desconfianza, contravalor que ya superó a su efecto positivo, es por eso que, este patrón del conflicto se relaciona con los procesos subyacentes que permiten transformar el conflicto, esto es que ante la existencia de una disputa en la cual estén en juego mis valores fundamentales, como lo es el caso de la confianza como valor intangible, lo que se concibe es que las partes pueden colaborar hacia la gestión, transformación y posible solución de la controversia, sería conveniente que mediante el acompañamiento de elementos generadores de confianza esta se visibiliza.

Estos elementos generadores son credibilidad, integridad, apertura y gestión de la comunicación y lealtad, y se presentan cuando:

- i. Se exterioriza la confianza, cuando se acude ante alguna institución a solicitar la impartición de justicia tradicional o alterna pública o privada como la mediación;

- ii. Se instaura en sí una litis o un proceso de mediación, en el que los participantes del conflicto deciden confiar y aceptan someterse al proceso o juicio, que da como resultado la generación de confianza entre las partes;

- iii. Se confía en el tercero interviniente llámese juez o facilitador, permitiendo que éste coadyube a poner fin a su disputa;

- iv. Acto anterior que conlleva a un re- sultado concreto, que sería la conclusión del juicio mediante una sentencia o en la mediación con un acuerdo en donde al aceptar tal veredicto o acuerdo, se tendrá la confianza plena y pura de que se materializarán al cumplir el contenido de estos.

\section{Patrón dominante de Expectativas}

La morfología del conflicto es sobre expectativas, ante este patrón (Redorta, 2014) manifiesta que el conflicto base que trata este patrón es esperanza/realidad, agrega la definición operativa consistente en que disputamos porque no se cumplió o se defraudó lo que uno esperaba del otro.

Respecto de este patrón dominante (Redorta, 2004) declara que la presencia de frustraciones está generalmente relacionada con las expectativas.

Por ello debemos examinar la base y el proceso de formación de tales expectativas. Luego procederemos a reducirlas para adecuarlas a la situación real. Como regla general, reconoceremos la legitimidad de las mismas. Por último, conviene controlar la agresividad que tiende a generar la frustración. Pues la confianza mutua puede ocurrir incluso en circunstancias en que las personas involucradas están abiertamente despreocupadas por el bienestar de los demás, siempre que las características de la situación sean tales que llevaría a esperar la confianza de uno a cumplirse; y la oportunidad y capacidad de comunicarse plenamente en un sistema de cooperación que define las responsabilidades mutuas (Deutsch, 1958, pág. 279). 
Para que proceso se lleve a cabo de forma asertiva y se materialice en una realidad tangible, es ineludible la estimulación como lo expresa (Redorta, 2004 p. 122) al decir que es necesaria la motivación para obtener algo que es muy importante, adecuando a nuestra investigación este contexto nos referimos a que el juez y facilitador juegan un papel fundamental en la solución de un conflicto, pues si las partes no confían plenamente en estos, el proceso fracasará. El profesional prestador de los servicios MASC, estará condenado al fracaso sino se confía en él (Gorjón, p. 81).

\section{Patrón dominante de Información}

Sobre este patrón la información es el patrón secundario del conflicto según (Redorta, 2014), y el conflicto básico consiste en conocer/ ignorar, y la definición operativa que formula sobre éste es que disputamos por algo que se dijo o no se dijo o que se entendió de forma distinta.

Las propuestas para este patrón dominante es que se apliquen las técnicas de comunicación. Es uno de los patrones más fáciles de afrontar, siempre que se disponga de la información relevante o que se trate de distorsiones de la información por excesiva, conviene utilizar la técnica del fact finding para objetivar los hechos.

Por lo que este patrón dominante reconoce la importancia de uno de los elementos generadores de confianza, ya que sin apertura y gestión de la comunicación no se configuraría este valor intangible, por ende, ante la simulación de alguno de los elementos se fundaría inmediatamente desconfianza.
La apertura y gestión de la comunicación implica la incorporación de comportamientos que muestren respeto, escuchar antes de todo, aclarar las expectativas, los cuales requieren colaboración entre carácter y competencia (Raya, 2018) además, de la forma de expresar sus emociones y pretensiones de manera no verbal y verbal, fomentar el diálogo; por ello es importante hacer énfasis en los puntos críticos que conviene explorar en este patrón dominante y así contribuir a la gestión y transformación del conflicto. Es evitando los errores en la percepción de lenguaje derivados de la diferencia del contexto social y cultural de los sujetos partícipes en una relación grupal y social, la ocultación de información y lo contrario a ésta, que conllevan al aprovechamiento de la relación afectiva para la procuración de intereses propios o de terceros, o el simple dolo, también con intenciones de beneficio directo o indirecto para la satisfacción de pretensiones.

\section{Patrón dominante Normativo}

El conflicto básico de la morfología de conflicto normativo es la conducta/norma, refiriéndose (Redorta, 2014) a que disputamos porque se incumple una norma legal o social.

En este patrón dominante conviene examinar la interpretación jurídica o social de las normas. De ese modo nos daremos cuenta de lo que supone cualquier desviación de la pauta generalmente aceptada.

En problemas grupales debemos plantearnos si existen minorías que desean cambios o reclaman influencia. 
El patrón dominante tiene relación con los puntos críticos de exploración que permiten la transformación del conflicto, es que ante el desconocimiento de la ley y la falta de credibilidad y desconfianza en las instituciones que componen a los tribunales tradicionales, las personas se privan de otro medio de impartición de justicia como lo son los MASC, tomando a la justicia tradicional como la primera instancia a la que el ciudadano recurre en lugar de los MASC que tienen el mismo fin. Provocando con ello que no se le dé la importancia debida a la confianza que merecen las instituciones del Estado; a que no se asista a los tribunales para que se les imparta justicia alternativa ni tradicional; además de que se genera una gran área de oportunidad para los abogados consultores - los litigantes a continuar con malas prácticas de privilegiar y llevar cualquier conflicto a juicio, tal y como lo exteriorizan (Gorjón Gómez \& Steele Garza, 2012) al decir que estos sólo piensan solo en su beneficio económico, dejando de lado beneficiar a la sociedad Por ello es menester contribuir a que el Poder Judicial se profesionalice y que conozca sólo los casos que las partes ya no puedan resolver por su cuenta, los litigantes no deben aprovecharse de las circunstancias del litigio para generar desconfianza a su cliente respecto al sistema judicial, las instituciones y a la formas de impartición de justicia.

\section{Patrón dominante de Estructural}

La definición operativa que expresa (Redorta, 2014) sobre este patrón es que disputamos por un problema cuya solución requiere largo tiempo, esfuerzo importante de muchos, o medios más allá de nuestras posibi- lidades personales. El conflicto base de este son medios/soluciones.

Las propuestas que enuncia para este patrón dominante es que en los conflictos estructurales no debemos actuar con precipitación.

La relación que tiene con nuestra problemática presentada, es que permite la transformación del conflicto, debiendo hacer énfasis en los procesos subyacente de este patrón, mostrando que de primera instancia no todas las personas tienen el libre derecho de acceso a la justicia, este derecho no está al alcance de todos, en toda sociedad y todo sistema jurídico se debe proveer a la población de modos de solucionar conflictos -judiciales-no alternos-y ejercer sus derechos; además, dichos modos deben estar al alcance de todos en condiciones de igualdad (Álvarez \& Highton, 1996) en (Gorjón Gómez \& López Sáenz, 2009, pág. 8), pues implica poseer ciertos atributos como poder, patrimonio, solvencia económica, entre otros factores indirectos de la persona como: sociales, políticos y culturales que impiden el libre ejercicio de este derecho.

Al mismo tiempo que los tribunales en el país, vistos como instituciones que administran e imparten justicia, no se encuentran plenamente capacitadas para ofrecer una vía alterna de justicia como lo hacen los MASC, pues no se tienen los suficientes recursos materiales y económicos para acercarse a la sociedad y dar a conocer, promover, y utilizar los métodos para dirimir sus conflictos, causando con ello que se prive de las cualidades que la mediación tiene; y por último 
que entre los individuos no se desarrolle la confianza como Valor intangible para vivir armónicamente en sociedad, por lo que si no se confía en los demás, mucho menos en instituciones y nuevas formas de impartir justicia.

\section{LA CONFIANZA COMO VALOR IN- TANGIBLE DE LOS MÉTODOS ALTERNA- TIVOS DE SOLUCIÓN DE CONFLICTOS}

Una vez conocida supralíneas la forma de cómo abordar el conflicto para su gestión, transformación y solución del mismo a través de la implementación de la confianza como valor intangible, también es de suma relevancia el conocer su origen epistémico que permita confabular una construcción concreta de intervención.

Hasta este momento como valor intangible ha sido estudiada sólo en los MASC particularmente en la mediación, por tal motivo nos limitaremos a describir este aspecto ya estudiado y probado mediante metodologías mixtas de investigación.

No existe hasta la fecha una clara estrategia para la culturización de la implementación de los MASC, es por lo que se admite considerar viable y ad hoc el redimensionarlos a través de sus valores intangibles como una estrategia de culturización, que nos permitirá traducir sus beneficios y su valor a la sociedad de una forma simple y contundente (Gorjón Gómez F. J., 2017, pág. 15).

Al considerarlos en una nueva clasificación las características y principios de los MASC se destaca su verdadera naturaleza que va más allá de su esencia, mostrando a los intangibles como beneficios traducidos en valor positivo.

En ese sentido (Edvinsson \& Malone, 2000) exponen que los intangibles son un valor sustentado en el capital intelectual que genera aprendizaje y adaptación, son las características distintivas que los diferencian de otros procesos y los hace útiles, atractivos, pero principalmente valiosos. Porque son una fuente de beneficios futuros sin sustancia física, generadores de valor que se forman a partir de la innovación, diseños organizativos únicos o prácticas de gestión (Lev, 2003) en (Gorjón Gómez F. J., 2017, págs. 18-19).

De una identificación total de 43 intangibles emanados de la Teoría del Valor Intangible de los MASC definida por y divulgada por primera vez en el año 2013 por (Gorjón Gómez F. J., págs. 48-54), pero que hasta el 2017 se formalizó en su obra "Los Intangibles de la Mediación y de los Métodos Alternos de Solución de Conflictos" (Gorjón Gómez F. J., 2017, págs. 80-82).

Para efectos del artículo en comento se analiza el conflicto causado por la desconfianza en los sistemas de impartición de justicia, desde diferentes enfoques que evidenciarán la trascendencia de este valor en la gestión, transformación y solución de conflictos, planteándolo desde la perspectiva de la problemática de estudio sobre la desconfianza en los sistemas de impartición de justicia mexicano con efectos más negativos particulares en la mediación como los MASC, el cual se sustentó en la herramienta (CAT) con el que 
se exhibió la existencia de patrones dominantes en la problemática presentada desde las instituciones impartidoras de justicia y dentro de la mediación, además de los procesos subyacentes de esos patrones (Redorta, 2014, pág. 117),. con el objetivo de centrar la intervención adecuada que permita la gestión, transformación y posible solución del mismo.

Para que la confianza pueda verse como vínculo conductor entre la ciudadanía y las instituciones encargadas en México de la impartición de justicia, y que los primeros asistan a ejercer su derecho humano de acceso a la justicia, ya sea para que se le ponga fin a una controversia o para que las personas mismas puedan solucionar sus conflictos y les permitan construir sociedades inclusivas de colaboración, democráticas, tolerantes y aseguradoras de los Derechos Humanos, pues causan y fomentan el diálogo, la solidaridad, la cooperación y la empatía, así como otros factores que robustecen las relaciones pacíficas entre las personas intervinientes de ese conflicto (Cabello Tijerina, y otros, 2017, pág. 66).

Distinguiendo las líneas de intervención de los conflictos, en la primera se encuadran aquellos sujetos que intervienen como "terceros", tomando la decisión de resolver el conflicto y ejecutar lo decidido, esta línea es la de heterocomposición. La segunda es la autocomposición que se basa en el supuesto de que las partes son los actores principales que resuelven el conflicto por su propia voluntad, y a pesar de que intervenga un tercero, la actuación de este es limitada y sin poder de decisión (Redorta, 2004, pág. 22).

\subsection{La Confianza como Valor Intangible de la Mediación}

En este sentido la Confianza como intangible de la mediación tiene diversos enfoques en relación al procedimiento de solución de conflictos alternos en el nuevo sistema acusatorio mexicano, este puede ser presentado por la confianza que sostienen las instituciones públicas y privadas para que se asista a estas a hacer valer el derecho humano de acceso a la justicia, la que el procedimiento genera en su conjunto a las partes, por la confianza que se le tiene al facilitador, derivada de la que él les genera y por la confianza plena sobre el cumplimiento inmediato del acuerdo de forma voluntaria, por consiguiente, un acuerdo de mediación sustentando y llevado a cabo a través de la confianza será más efectivo y, para el caso de los acuerdos de tracto sucesivo, será más eficiente asegurando su observancia, en razón de sus intereses personales para que se ejecute, en tiempo y debidamente (Covey \& Merrill, 2010) (Brogan \& Smith, 2013) en (Gorjón Gómez F. J., 2017, pág. 80).

Además, la confianza es un factor de éxito en el procedimiento de solución del conflicto, ya que, si las partes no confían en el mediador, estará destinado al fracaso (Villa \& Caperán, 2010) en (Gorjón Gómez F. J., 2017, pág. 81).

Recordemos entonces como lo manifiesta (Gorjón Gómez F. J., págs. 15,63), que los intangibles de los MASC son una estrategia de comunicación, por lo tanto, lograr comunicar los intereses asertivamente ante la existencia de un conflicto, tendrá un efecto positivo y en consecuencia la solución del conflicto y 
generará éxito en la tarea de la culturización que se propone y su credibilidad en el proceso de transformación de los conflictos, así como transmitir el valor positivo y su impacto social a todos los sectores de la ciudadanía. Una vez expuesta a la confianza como intangible, el origen y su dimensionalidad en los MASC, es importante puntualizar que existen teorías sobre la confianza del ser humano a quien conciben como persona física o moral en los diferentes sectores sociales que se desarrolla y en otros campos de estudio afines, enfrentándose a conflictos, en los cuales se ignora las herramientas mínimas que aportan a su gestión, trasformación y solución de estos, por lo que nos enfocaremos hacia aquellas que más robustecieron la gestión, transformación de la problemática social planteada, resultantes de la ejecución del CAT.

En el mismo sentido dentro de los MASC, Stephen B. Goldberg (2005) en un intento por comprender los factores que preceden el éxito de la mediación, realizó una investigación con encuestas a 30 mediadores. Este estudio reveló que el éxito de la mediación dependía de la construcción del rapport, es decir, la construcción de "una relación de mutua comprensión, empatía y confianza entre las partes". Posteriormente 2007 (Goldberg \& Shaw, pág. 366) llevaron a cabo un segundo estudio que contó con la participación de 216 mediadores de los cuales 152 eran abogados. El objetivo principal del mismo fue mejorar la formación de los mediadores a través de la comprensión de los factores que explican el éxito de la Mediación. Dado que las aportaciones de los mediadores fueron recogidas en el primer estudio de
Goldberg, en el segundo se recogieron las aportaciones de las partes. Los resultados demostraron un total de 20 habilidades que ambos autores convinieron clasificar en tres grupos, entre ellos la confianza o llamado "fomento de la confianza". Dichos autores, aportaron a la evidencia empírica un silogismo como resultado de dos nuevos estudios que fueron acometidos de forma paralela. Uno de ellos fue que supeditaron el éxito de la mediación a la construcción de una relación de confianza (Goldberg, Shaw, \& Brett, 2009) en (Riera Adrover, 2018, págs. 55,181) para la gestión, transformación y solución de conflictos en el ámbito jurídico.

En el marco del liderazgo y emprendimiento Stephen (Covey \& Merrill) presentan a la confianza como un sentimiento hacia tu persona o un sentimiento hacia otra individuo ya sea física o moral, este efecto se conoce como las cinco ondas de la confianza denominadas: 1. confianza en uno mismo, 2. confianza en las relaciones, 3. confianza empresarial, 4. confianza del mercado y 5. confianza social en (Gorjón Gómez F. J., 2017, pág. 81) la cual permite la generación de relaciones de confianza con base en la gestión y trasformación de conflictos que se presentan entre todos los sujetos que emplean el liderazgo y emprendimiento como un modus vivendi.

Esta teoría de las cinco ondas de la confian$z a$, explica que los elementos que forjan la confianza son Credibilidad, Remediar los errores, Integridad, Intención, Capacidad, Resultados, Ser sinceros, Mostrar respeto, Ser cristalinos, Demostrar lealtad, Producir resultados, Enfrentarse a la realidad, Aclarar las expectativas, Ejercer la responsabi- 
lidad, Escuchar antes de todo, Mantener los compromisos, Transmitir confianza y Mejora manifestándose de adentro hacia afuera dependiendo la onda de que se trate (Covey S. M., 2011).

En la primera onda basada en la autoconfianza el principio clave de esta es la credibilidad, la cual a su vez se basa en cuatro pilares, uno de ellos es la integridad. La segunda onda de la confianza en la relaciones, se basa en un comportamiento coherente y está influenciada por 13 comportamientos clave que tienen los líderes con alto grado de confianza, los cuales se pueden aprender y aplicar, uno de ellos es la lealtad, este deriva del carácter; para nuestro último elemento, descubrimos a la apertura de la comunicación concentrada en algunos comportamientos como: mostrar respeto, escuchar antes de todo, aclarar las expectativas, los cuales requieren colaboración entre carácter y competencia (Raya, 2018).

En el ámbito de la economía, las teorías de prosperidad nacional consienten crear relaciones de confianza entre diversos actores de la sociedad civil, públicos y privados, permitiendo identificar posibles conflictos en actividades económicas Fukuyama (Trust: the social virtues and the creation of prosperity, 1995, pág. 26) manifiesta al respecto que es la esperanza que emerge dentro de la comunidad de honradez y conducta cooperativa, basada en normas comunes, sobre la parte de otros miembros de esa comunidad. Es decir, Fukuyama (1995) y su teoría llamada Tesis de la Confianza del año 1995 pretendía enlazar la cultura con la economía explicando la influencia de la primera en la segunda; en ese sentido lo más importante es el nivel de confianza existente en la sociedad para delegar determinadas actividades económicas. Puede suceder que los agentes económicos tengan relación de confianza mutua, dando como resultado grandes compañías y una enorme sociedad civil. O puede suceder que exista una ausencia de confianza que provoque una pobre realización económica. El autor clasificó a los países según la confianza en la sociedad civil, con base en el grado que las sociedades tenían para subordinar sus intereses a la comunidad en alta y baja confianza (Sanmartín, pág. 42) siendo los elementos que la componen el Reconocimiento, Religión, Justicia, Prestigio y Honor.

Cuando Fukuyama (1995) distingue entre una sociedad de alta confianza y una de baja confianza, indica que estos no son dos destinos inmutables, sino susceptibles de ser transformados mediante el cultivo de la confianza en (Picker, 2012, pág. 2).

\section{CONCLUSIONES}

Como se pudo evidenciar, al justificar teórica y estadísticamente los niveles de confianza, de percepción existente de confianza interpersonal y la manifestada hacia las instituciones encargadas en México de la impartición de justicia tradicional y alternativa que sustentan la problemática descrita; y que mediante la aplicación del dispositivo CAT en este artículo, logramos exponer que los patrones dominantes en nuestro problema de estudio son de valores, información, expectativas, normativo, estructural; los cuales con su presencia fortalecen nuestra 
investigación de "Los elementos generadores de Confianza como Valor intangible en la mediación" además, que proporcionan rigor científico a la misma.

Estos cinco patrones dominantes, fueron determinantes en el desarrollo de la investigación, por lo que coincidimos plenamente con (Redorta) en que se debe trabajar sobre ellos para la posible solución del conflicto expuesto; todo esto con base en los puntos críticos detallados en este artículo, así como sobre las diversas estrategias que propone el conflictólogo para la transformación de este.

Si bien es cierto que los elementos generadores de confianza dentro de los patrones dominantes del conflicto planteado sirven ahora como indicadores, también debemos coadyubar al desarrollo de futuras investigaciones que permitan contribuir a la solución de la problemática planteada y que sirva como antecedente para la ciudadanía a que confíen en sí mismos para la solución de sus propios conflictos, los que surgen de su relación como ente social y los que versan ante las instituciones encargadas en México de la impartición de justicia tradicional y alternativa, llámese juicio, conciliación o como en este caso la mediación, donde la Confianza como Valor intangible apoye a dar solución a un conflicto, y mejor aún, se pueda palpar la materialización de la voluntad acordada por las partes intervinientes mediante el cumplimiento del acuerdo resultante de esa mediación asertiva, que favorecerá a la generación de sociedades pacíficas, felices, solidarias y cooperativas que fomenten el diálogo.

\section{TRABAJOS CITADOS}

Álvarez, G. S., \& Highton, E. I. (1996). Mediación y Justicia. Buenos Aires: Desalma.

Brogan, C., \& Smith, J. (2013). La fórmula del éxito. Barcelona: Gestión.

Cabello Tijerina, P. A., Carmona Valdés, S. E., Gorjón Gómez, F. J., Iglesias Ortuño, E., Sáenz López, K. A., \& y Vázquez Gutiérrez, R. L. (2017). Cultura de paz. México, Patria

Covey, S. M. (2011). El factor de la confianza. El valor que lo cambia todo. Buenos Aires: Paidós.

Covey, S. M., \& Merrill, R. R. (2008). La velocidad de la confianza. El valor que lo cambia todo Buenos Aires. Paidós.

Creswell, J. W., \& Plano Clark, V. L. (2007). Designing and conducting Mixed Methods Research. California: Sage Publications.

Deutsch, M. (1958). Trust and Suspicion. The Journal of Conflict Resolution, pp. 265-279. Recuperado en: doi:https://doi.org/10.1177/002200275800200401

Edvinsson, L., \& y Malone, M. (2000). El capital intelectual. Como identificar y calcular el valor de los recursos intangibles de una empresa. Barcelona: Gestión.

Fisas Armengol, V. (1987). Introducción al estudio de la paz y de los conflictos. Barcelona: Lerna.

Fukuyama, F. (1995). Trust: the social virfues and the creation of prosperity. Nueva York: The Free Press. Recuperado en: https://doi.org/10.1177/089692059602200108

Gobernación, S. d. (2012). Encuesta Nacional sobre Cultura Política y Prácticas Ciudadanas. Recuperado el 08 de marzo de 2018 en: http://www.encup.gob.mx/work/models/Encup/Resource/69/1/images/Resultados-Quinta-ENCUP-2012.pdf

Goldberg, S. B. (2005). The secrets of successful mediators. Negotiation Journal, 365-376. Recuperado en: https: doi:10.1111/j.0748-4526.2005.00069.x

Goldberg, S.B., \& y Shaw, M. L. (2007). The secrets of successful (and unsuccessful mediators) continued: Studies two and three. Negotiation Journal, 393-418. Recuperado en: https:doi:10.1111/j.1571-9979.2007.00152.x 
Goldberg, S.B., Shaw, M. L., \& Brett, J. M. (2009). What difference does a robe make? Comparing mediators with and without prior judicial experience: Research reports. Negotiation Journal, 277-305. Recuperado en: https:doi :10.1111/j.1571-9979.2009.00227.x

Gorjón Gómez, F. J. (septiembre de 2013). El Valor Intangible de los Métodos Alternos de Solución de Conflictos "Estrategia de culturización de los MASC en el marco de la Reforma Procesal Penal". Revista Nuevo Sistema de Justicia Penal, IV (6), pp. 48-55. Recuperado en: https:// www.gob.mx/cms/uploads/attachment/file/53035/Revista_NSJP_VI.pdf

(2017). Mediación, su valor intangible y efectos operativos. Una visión Integradora de los Métodos Alternos de Solución de Conflictos. México: Tirant Lo Blanch.

Gorjón Gómez, F. J., \& López Sáenz, K. A. (2009). Métodos Alternos de Solución de Controversias Enfoque educativo por competencias. México: Patria.

Gorjón Gómez, F. J., \& Steele Garza, J. G. (2012). Métodos alternativos de solución de conflictos. México, D.F.:Oxford.

Instituto Nacional Electoral [INE]. (2015). Informe País sobre la calidad de la ciudadanía en México. México INE.

Lagos, M. (2015). Latinobárometro 1995-2015 Opinión Pública Latinoamericana. Recuperado el 21 de 05 de 2018 en: http://www.latinobarometro.org/LATDocs/ F00005085-INFORME_LB_LA_CONFIANZA_1995_2015. pdf

Lev, B. (2003). Intangibles. Mediación, gestión e información. Bilbao: Deusto.

Moreno, A. (2005). Nuestros valores, los mexicanos en México y en Estados Unidos al inicio del siglo XXI. México, D.F.:Grupo Financiero Banamex.

Picker, H. (2012). La confianza. CEC internacional, 9. Recuperado en que fecha en: http://www.cecinternational. com/wp-content/uploads/2015/04/16-La-confianzaNT-v1-.pdf

Raya, A. (2018). Andrés Raya. Management is doing things right, leadership is doing thw right things. Recuperado en que fecha en: http://andresraya.com/el-poder-y-la- velocidad-de-la-confianza/

Redorta, J. (2004). Cómo analizar los conflictos. La tipología de conflictos como herramienta de mediación. México, Paidós.

(2014). Análisis de los conflictos por patrones: La nueva herramienta CAT ${ }^{\odot}$. Democracia Digital e Governo Eletrônico, pp. 310-323. Obtenido de Recuperado en que fecha en: http://www.buscalegis.ufsc.br/ revistas/files/journals/3/articles/34338/submission/original/34338-45979-1-SM.doc

-(2014). Entender el conflicto. La forma como herramienta. Barcelona: Paidós.

(2016). Conflict management. Ciencia aplicada a la gestión del conflicto. Obtenido de Recuperado en que fecha en: https://books.google.com.mx/ books?id=ZOIsDwAAQBAJ\&pg=PT61\&lpg =PT61\&d$q=$ ser,+ seguir,+ conseguir $+y+$ mantener $+\% 2 B+$ redorta\&source =b|\&ots=Z_cq2Ar_4X\&sig=qgMSR7cvG3NleYDb6qy0Jr6RsvM\&hl=es\&sa=X\&ved=2ahUKEwiMqMD1I-TeAhURQq0KHSs-CqgQ6AEwCXoECAgQAQ\#$\mathrm{v}=$ onepage $\& \mathrm{q}=\mathrm{ser} \% 2 \mathrm{C} \%$

Riera Adrover, J. A. (2018). La generación de confianza en la práctica de la mediación familiar: ¿por qué las partes confían en los mediadores? Tesis Doctoral. Recuperado en que fecha en: https://www.tesisenred.net/bitstream/ handle $/ 10803 / 666349 /$ tjaralde1.pdf?sequence=1\&isAllowed=y

Sanmartín, I. (s.f.). Biblioteca Saavedra Fajardo de pensamiento Políitico hispánico. Obtenido de Recuperado en que fecha en: http://www.saavedrafajardo.org/

\section{Jazmín Flores}

Doctora en MSC por la Universidad Autónoma de Nuevo León, México. Máster en Derecho Constitucional y Gobernabilidad. Profesora de MSC y Cultura de Paz por la Universidad Autónoma de Nuevo León, México. Email: jazmin_7_7@hotmail.com https://orcid. org/0000-0001-8197-6782 
